




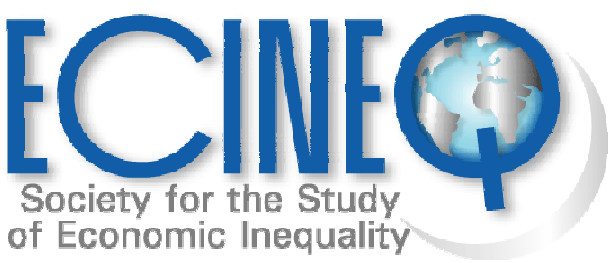

ECINEQ 2010-160

March 2010

of Economic Inequality

www.ecineq.org

\title{
A class of poverty measures induced by the dual decomposition of aggregation functions
}

\author{
José Luis García-Lapresta* \\ University of Valladolid
}

Casilda Lasso de la Vega

University of the Basque Country

\author{
Ricardo Alberto Marques Pereira \\ Università degli Studi di Trento \\ Ana Marta Urrutia \\ University of the Basque Country
}

\begin{abstract}
In this paper we introduce a new family of poverty measures for comparing and ordering social situations. The aggregation scheme of these poverty measures is based on the exponential means. The poverty measures introduced satisfy interesting properties and their dual decomposition provides a factorization of the proposed indices into three underlying factors: incidence, intensity and inequality among the poor.
\end{abstract}

Keywords: economic indices, welfare, inequality, poverty decomposition, aggregation functions, exponential means, dual decomposition.

JEL classification: D63, I32.

Address of correspondence: lapresta@eco.uva.es 


\section{Introduction}

In the economic literature there exists a large variety of economic indices (inequality, poverty, social development, gross domestic product, health, welfare, etc.) for comparing and ranking social situations across populations (see, for instance, Sen [15], Dagum [7], and Chakravarty and Muliere $[5,6]$ ). A thorough survey of the literature on inequality, poverty, and welfare can be found in Chakravarty $[3,4]$. The main difference between inequality or welfare and poverty lies in their focus. Whereas inequality and welfare measurement involves the whole population, the concern of poverty is focused on those who are worse-off.

Specifically, any poverty measure includes a method to identify the poor and an aggregative measure. The identification step requires the specification of a poverty line that splits society into two groups: the poor and the non-poor people. With respect to the aggregation stage, according to Sen [14], any poverty index should be sensitive to the number of people below the poverty line, to the extent of the income short-fall of the poor from the poverty line, and to the exact pattern of the income distribution of the poor. In other words, every poverty measure should be expressed as a function of these three poverty indicators, showing the incidence, the intensity and the inequality of the poverty, respectively. Poverty changes can be more meaningful and easily understandable if poverty indices can be decomposed into these underlying contributing factors.

In this paper we introduce a new family of poverty measures based on the exponential means in order to aggregate the normalized income gaps of the poor individuals. The exponential means have already been used to define inequality and welfare measures. However, to our knowledge, no relative poverty indices have been proposed involving the exponential means.

The family of poverty measures introduced in this paper has three interesting features. Firstly, all the poverty measures proposed are invariant under changes in the unit in which income is measured. Secondly, given a poverty line and a set of poor individuals, when all poor individuals receive the same amount of extra income the reduction in the overall poverty level depends only on that extra amount. Finally, all the members of the new family of poverty measures are more sensitive to changes at the lower end of the income profile, that is, to what happens to the poorest of the poor. In addition, the family parameter can be interpreted as a measure of this sensitivity. None of the indices introduced in the literature are able to capture these three issues together.

With respect to the decomposition of these indices in the three underlying contribution factors, we propose to use the dual decomposition of aggregations function into a self-dual core and anti-self-dual remainder as applied to the family of exponential means (see García-Lapresta 
and Marques Pereira [9]). We show that these two terms can be interpreted as measures of the intensity and of the inequality among the poor, respectively. This decomposition allows us to know if increasing poverty is due to more people becoming poor, or increasing deprivation of the poor, or because income short-fall below the poverty line have become more unequal, or some combination of the above. Therefore, the decomposition proposed can be useful to have a better understanding of the measurement of poverty, particularly in a dynamical scenario.

The paper is organized as follows. Section 2 introduces basic notation and properties of aggregation functions. Section 3 is devoted to summarize the dual decomposition of an aggregation function into a self-dual core and an associated anti-self-dual remainder. In Section 4 we review the class of exponential means and discuss their dual decomposition. In Section 5 we introduce our proposal for measuring poverty using exponential means and propose a decomposition of the indices into incidence, intensity, and inequality. An illustrative example is described. Finally, Section 6 contains some concluding remarks.

\section{Aggregation functions}

In this section we present notation and basic definitions regarding aggregation functions on $[0,1]^{n}$, with $n \in \mathbb{N}$ and $n \geq 2$ throughout the text.

Notation. Points in $[0,1]^{n}$ will be denoted by means of boldface characters: $\boldsymbol{x}=\left(x_{1}, \ldots, x_{n}\right), \mathbf{1}=(1, \ldots, 1), \mathbf{0}=(0, \ldots, 0)$. For $x \in[0,1]$, we have $x \cdot \mathbf{1}=(x, \ldots, x)$. Given $\boldsymbol{x}, \boldsymbol{y} \in[0,1]^{n}$, by $\boldsymbol{x} \geq \boldsymbol{y}$ we mean $x_{i} \geq y_{i}$ for every $i \in\{1, \ldots, n\}$; by $\boldsymbol{x}>\boldsymbol{y}$ we mean $\boldsymbol{x} \geq \boldsymbol{y}$ and $\boldsymbol{x} \neq \boldsymbol{y}$. Given $\boldsymbol{x} \in[0,1]^{n}$, with $\left(x_{(1)}, \ldots, x_{(n)}\right)$ we denote the increasing ordered version of $\boldsymbol{x}$, i.e., $x_{(i)}$ is the $i$-th lowest number of $\left\{x_{1}, \ldots, x_{n}\right\}$. Moreover, $x_{*}=x_{(1)}=\min \left\{x_{1}, \ldots, x_{n}\right\}$ and $x^{*}=x_{(n)}=\max \left\{x_{1}, \ldots, x_{n}\right\}$. Given a permutation on $\{1, \ldots, n\}$, i.e., a bijection $\sigma:\{1, \ldots, n\} \longrightarrow\{1, \ldots, n\}$, with $\boldsymbol{x}_{\sigma}$ we denote $\left(x_{\sigma(1)}, \ldots, x_{\sigma(n)}\right)$.

We begin by defining standard properties of real functions on $[0,1]^{n}$. On this, see Fodor and Roubens [8], Calvo et al. [2], Beliakov et al. [1], García-Lapresta and Marques Pereira [9] and Grabisch et al. [10].

Definition 2.1 Let $A:[0,1]^{n} \longrightarrow \mathbb{R}$ be a function.

1. A is idempotent if for every $x \in[0,1]$ :

$$
A(x \cdot \mathbf{1})=x .
$$

2. $A$ is symmetric if for every permutation $\sigma$ on $\{1, \ldots, n\}$ and every $\boldsymbol{x} \in[0,1]^{n}$ :

$$
A\left(\boldsymbol{x}_{\sigma}\right)=A(\boldsymbol{x}) .
$$


3. $A$ is monotonic if for all $\boldsymbol{x}, \boldsymbol{y} \in[0,1]^{n}$ :

$$
\boldsymbol{x} \geq \boldsymbol{y} \Rightarrow A(\boldsymbol{x}) \geq A(\boldsymbol{y}) .
$$

4. $A$ is strictly monotonic if for all $\boldsymbol{x}, \boldsymbol{y} \in[0,1]^{n}$ :

$$
\boldsymbol{x}>\boldsymbol{y} \Rightarrow A(\boldsymbol{x})>A(\boldsymbol{y}) .
$$

5. A is compensative if for every $\boldsymbol{x} \in[0,1]^{n}$ :

$$
x_{*} \leq A(\boldsymbol{x}) \leq x^{*} .
$$

6. $A$ is self-dual if for every $\boldsymbol{x} \in[0,1]^{n}$ :

$$
A(\mathbf{1}-\boldsymbol{x})=1-A(\boldsymbol{x})
$$

7. $A$ is anti-self-dual if for every $\boldsymbol{x} \in[0,1]^{n}$ :

$$
A(\mathbf{1}-\boldsymbol{x})=A(\boldsymbol{x}) .
$$

8. A is invariant for translations if for all $t \in[-1,1]$ and $\boldsymbol{x} \in[0,1]^{n}$ :

$$
A(\boldsymbol{x}+t \cdot \mathbf{1})=A(\boldsymbol{x})
$$

whenever $\boldsymbol{x}+t \cdot \mathbf{1} \in[0,1]^{n}$.

9. $A$ is stable for translations if for all $t \in[-1,1]$ and $\boldsymbol{x} \in[0,1]^{n}$ :

$$
A(\boldsymbol{x}+t \cdot \mathbf{1})=A(\boldsymbol{x})+t
$$

whenever $\boldsymbol{x}+t \cdot \mathbf{1} \in[0,1]^{n}$.

Consider the binary relation $\succcurlyeq$ on $[0, \infty)^{n}$ given by

$$
\boldsymbol{x} \succcurlyeq \boldsymbol{y} \Leftrightarrow \sum_{i=1}^{n} x_{i}=\sum_{i=1}^{n} y_{i} \text { and } \sum_{i=1}^{k} x_{(i)} \leq \sum_{i=1}^{k} y_{(i)} \text { for every } k \in\{1, \ldots, n-1\} .
$$

(10) $A$ is $S$-convex if for all $\boldsymbol{x}, \boldsymbol{y} \in[0,1]^{n}$ :

$$
\boldsymbol{x} \succcurlyeq \boldsymbol{y} \Rightarrow A(\boldsymbol{x}) \geq A(\boldsymbol{y}) .
$$

(11) $A$ is $S$-concave if for all $\boldsymbol{x}, \boldsymbol{y} \in[0,1]^{n}$ :

$$
\boldsymbol{x} \succcurlyeq \boldsymbol{y} \Rightarrow A(\boldsymbol{x}) \leq A(\boldsymbol{y}) .
$$


Let now $\left\{A^{(k)}\right\}_{k \in \mathbb{N}}$ be a sequence of functions, where $A^{(k)}:[0,1]^{k} \longrightarrow \mathbb{R}$ and $A^{(1)}(x)=x$ for every $x \in[0,1]$.

(12) $\left\{A^{(k)}\right\}_{k \in \mathbb{N}}$ is invariant for replications if for all $\boldsymbol{x} \in[0,1]^{n}$ and any number of replications $m \in \mathbb{N}$ of $\boldsymbol{x}:$

$$
A^{(m n)}(\overbrace{\boldsymbol{x}, \ldots, \boldsymbol{x}}^{m})=A^{(n)}(\boldsymbol{x})
$$

(13) $\left\{A^{(k)}\right\}_{k \in \mathbb{N}}$ is decomposable (or separable) if, for any given subset of variables, every variable in the subset can have its value replaced by the partial aggregated value of the subset without altering the overall aggregated value of the full set of variables, for instance if

$$
\begin{aligned}
& A^{(n)}\left(x_{1}, \ldots, x_{m}, x_{m+1}, \ldots, x_{n}\right)= \\
& =A^{(n)}\left(A^{(m)}\left(x_{1}, \ldots, x_{m}\right), \ldots, A^{(m)}\left(x_{1}, \ldots, x_{m}\right), x_{m+1}, \ldots, x_{n}\right),
\end{aligned}
$$

for all $\boldsymbol{x} \in[0,1]^{n}$ and $m \leq n$.

Definition 2.2 A function $A:[0,1]^{n} \longrightarrow[0,1]$ is called an $n$-ary aggregation function if it is monotonic and satisfies $A(\mathbf{0})=0$ and $A(\mathbf{1})=1$. For the sake of simplicity, the $n$-arity is omitted whenever it is clear from the context. An aggregation function is said to be strict if it is strictly monotonic.

Self-duality and stability for translations are important properties of aggregation functions. In turn, anti-self-duality and invariance for translations are incompatible with the defining properties of aggregation functions, namely with the boundary conditions $A(\mathbf{0})=0$ and $A(\mathbf{1})=1$. Nevertheless, anti-self-duality and invariance for translations play an important role in this paper in so far as they are properties of important functions associated with aggregation functions, as we shall discuss later. The following are standard facts concerning aggregation functions (see García-Lapresta and Marques Pereira [9]).

Proposition 2.3 Let $A:[0,1]^{n} \longrightarrow[0,1]$ be an aggregation function.

1. A is idempotent if and only if $A$ is compensative.

2. If $A$ is strict, then $A(\boldsymbol{x})=0$ if and only if $\boldsymbol{x}=\mathbf{0}$, and $A(\boldsymbol{x})=1$ if and only if $\boldsymbol{x}=\mathbf{1}$.

3. If $A$ is stable for translations, then $A$ is idempotent. 


\section{The self-dual core and the associated remainder}

In this section we briefly summarize the decomposition of an aggregation function in the selfdual core and the associated remainder included in García-Lapresta and Marques Pereira [9]. First we introduce the concepts of self-dual core and anti-self-dual remainder of an aggregation function, establishing which properties are inherited in each case from the original aggregation function. Particular emphasis is given to the properties of stability for translations (self-dual core) and invariance for translations (anti-self-dual remainder).

Definition 3.1 Let $A:[0,1]^{n} \longrightarrow[0,1]$ be an aggregation function. The aggregation function $A^{*}:[0,1]^{n} \longrightarrow[0,1]$ defined as

$$
A^{*}(\boldsymbol{x})=1-A(\mathbf{1}-\boldsymbol{x})
$$

is known as the dual of the aggregation function $A$.

Clearly, $\left(A^{*}\right)^{*}=A$, which means that dualization is an involution. An aggregation function $A$ is self-dual if and only if $A^{*}=A$.

Proposition 3.2 Let $A:[0,1]^{n} \longrightarrow[0,1]$ be an aggregation function. The dual $A^{*}$ inherits from the aggregation function $A$ the properties of idempotency (hence, compensativeness), symmetry, strict monotonicity, continuity, self-duality, anti-self-duality, invariance and stability for translations, invariance for replications and decomposability, whenever $A$ has these properties. In addition, $A^{*}$ is $S$-convex (resp. $S$-concave) whenever $A$ is $S$-concave (resp. $S$-convex).

Proof. We only prove the last property: if $A$ is $S$-concave, then $A^{*}$ is $S$-convex (the previous ones are mechanical). It is easy to check that $\boldsymbol{x} \succcurlyeq \boldsymbol{y} \Rightarrow \mathbf{1}-\boldsymbol{x} \succcurlyeq \mathbf{1}-\boldsymbol{y}$. If $A$ is $S$-concave, then from $\boldsymbol{x} \succcurlyeq \boldsymbol{y}$ we have $A(\boldsymbol{x}) \leq A(\boldsymbol{y})$ and also $A(\mathbf{1}-\boldsymbol{x}) \leq A(\mathbf{1}-\boldsymbol{y})$. Consequently,

$$
A^{*}(\boldsymbol{x})=1-A(\mathbf{1}-\boldsymbol{x}) \geq 1-A(\mathbf{1}-\boldsymbol{y})=A^{*}(\boldsymbol{y}),
$$

i.e., $A^{*}$ is $S$-convex.

\subsection{The core}

Aggregation functions are not in general self-dual. However, a self-dual aggregation function can be associated to any aggregation function in a simple manner. The construction of the so-called self-dual core of an aggregation function $A$ is as follows. 
Definition 3.3 Let $A:[0,1]^{n} \longrightarrow[0,1]$ be an aggregation function.

The function $\widehat{A}:[0,1]^{n} \longrightarrow[0,1]$ defined by

$$
\widehat{A}(\boldsymbol{x})=\frac{A(\boldsymbol{x})+A^{*}(\boldsymbol{x})}{2}=\frac{A(\boldsymbol{x})-A(\mathbf{1}-\boldsymbol{x})+1}{2} .
$$

is called the core of the aggregation function $A$.

Notice that $\widehat{A}$ is clearly an aggregation function, verifying the boundary conditions $\widehat{A}(\mathbf{0})=0$, $\widehat{A}(\mathbf{1})=1$ and monotonicity. Since $\widehat{A}$ is self-dual, we say that $\widehat{A}$ is the self-dual core of the aggregation function $A$.

It is interesting to note that the self-dual core reduces to the arithmetic mean in the simple case of $n=2$ but not in higher dimensions.

The following results ${ }^{1}$ can be found in in García-Lapresta and Marques Pereira [9].

Proposition 3.4 An aggregation function $A:[0,1]^{n} \longrightarrow[0,1]$ is self-dual if and only if $\widehat{A}(\boldsymbol{x})=A(\boldsymbol{x})$ for every $\boldsymbol{x} \in[0,1]^{n}$.

Proposition 3.5 The self-dual core $\widehat{A}$ inherits from the aggregation function $A$ the properties of idempotency (hence, compensativeness), symmetry, strict monotonicity, continuity, stability for translations and invariance for replications, whenever $A$ has these properties.

\subsection{The remainder}

We now introduce the anti-self-dual remainder $\widetilde{A}$, which is simply the difference between the original aggregation function $A$ and its self-dual core $\widehat{A}$.

Definition 3.6 Let $A:[0,1]^{n} \longrightarrow[0,1]$ be an aggregation function.

The function $\widetilde{A}:[0,1]^{n} \longrightarrow \mathbb{R}$ defined by $\widetilde{A}(\boldsymbol{x})=A(\boldsymbol{x})-\widehat{A}(\boldsymbol{x})$, that is

$$
\widetilde{A}(\boldsymbol{x})=\frac{A(\boldsymbol{x})-A^{*}(\boldsymbol{x})}{2}=\frac{A(\boldsymbol{x})+A(\mathbf{1}-\boldsymbol{x})-1}{2},
$$

is called the remainder of the aggregation function $A$.

Notice that $\widetilde{A}$ is anti-self-dual. For this reason we say that $\widetilde{A}$ is the anti-self-dual remainder of the aggregation function $A$. Clearly, $\widetilde{A}$ is not an aggregation function. In particular, $\widetilde{A}(\mathbf{0})=$

\footnotetext{
${ }^{1}$ Excepting that invariance for replications is inherited by the core (the proof is immediate).
} 
$\widetilde{A}(\mathbf{1})=0$ violates the boundary conditions and implies that $\widetilde{A}$ is either non monotonic or everywhere null. Moreover, $-0.5 \leq \widetilde{A}(\boldsymbol{x}) \leq 0.5$ for every $\boldsymbol{x} \in[0,1]^{n}$.

The following results ${ }^{2}$ can be found in in García-Lapresta and Marques Pereira [9].

Proposition 3.7 An aggregation function $A:[0,1]^{n} \longrightarrow[0,1]$ is self-dual if and only if $\widetilde{A}(\boldsymbol{x})=0$ for every $\boldsymbol{x} \in[0,1]^{n}$.

Proposition 3.8 The remainder $\widetilde{A}$ inherits from the aggregation function $A$ the properties of symmetry, continuity, $S$-convexity, $S$-concavity and invariance for replications, whenever $A$ has these properties.

Summarizing, every aggregation function $A$ decomposes additively $A=\widehat{A}+\widetilde{A}$ in two components: the self-dual core $\widehat{A}$ and the anti-self-dual remainder $\widetilde{A}$, where only $\widehat{A}$ is an aggregation function. The so-called dual decomposition $A=\widehat{A}+\widetilde{A}$ clearly shows some analogy with other algebraic decompositions, such as that of square matrices and bilinear tensors into their symmetric and skew-symmetric components.

Since $A^{*}=\widehat{A}-\widetilde{A}$, we have a new decomposition: $A=A^{*}+2 \widetilde{A}$.

The following result concerns two more properties of the anti-self-dual remainder based directly on the definition $\widetilde{A}=A-\widehat{A}$ and the corresponding properties of the self-dual core (see García-Lapresta and Marques Pereira [9]).

Proposition 3.9 Let $A:[0,1]^{n} \longrightarrow[0,1]$ be an aggregation function.

1. If $A$ is idempotent, then $\widetilde{A}(x \cdot \mathbf{1})=0$ for every $x \in[0,1]$.

2. If $A$ is stable for translations, then $\widetilde{A}$ is invariant for translations.

These properties of the anti-self-dual remainder are suggestive. The first statement applies to the class of idempotent aggregation functions. In such case, self-dual cores are idempotent and therefore anti-self-dual remainders are null on the main diagonal. The second statement applies to the subclass of stable aggregation functions. In such case, self-dual cores are stable and therefore anti-self-dual remainders are invariant for translations. In other words, if the aggregation function $A$ is stable for translations, the value $\widetilde{A}(\boldsymbol{x})$ does not depend on the average value of the $\boldsymbol{x}$ coordinates, but only on their numerical deviations from that average value. These properties of the anti-self-dual remainder $\widetilde{A}$ suggest that it may give some indication on the dispersion of the $\boldsymbol{x}$ coordinates.

\footnotetext{
${ }^{2}$ Excepting that invariance for replications is inherited by the remainder (the proof is immediate).
} 


\section{Exponential means}

Quasiarithmetic means are aggregation functions that satisfy interesting properties. They were simultaneously characterized in 1930 by Kolmogoroff [11] and Nagumo [13] (see also Fodor and Roubens [8, pp. 112-114]) through five properties: continuity, idempotency, symmetry, strict monotonicity and decomposability. Exponential means are quasiarithmetic means that are stable for translations. Thus, their cores are also stable for translations, and their anti-selfdual remainders are invariant for translations, joint with other interesting properties. This is the reason why we consider these functions for defining appropriate measures in our setting.

Definition 4.1 Let $A:[0,1]^{n} \longrightarrow[0,1]$ be an aggregation function. We say that $A$ is a quasiarithmetic mean if there exists an order automorphism (bijective and increasing function) $\varphi:[0,1] \longrightarrow[0,1]$ such that

$$
A(\boldsymbol{x})=\varphi^{-1}\left(\frac{\varphi\left(x_{1}\right)+\cdots+\varphi\left(x_{n}\right)}{n}\right)
$$

where $\varphi$ is said to generate the quasiarithmetic mean $A$.

Exponential means are the quasiarithmetic means generated by the order automorphisms $\varphi_{\alpha}$, $\alpha \neq 0$

$$
\varphi_{\alpha}(x)=\frac{e^{\alpha x}-1}{e^{\alpha}-1}
$$

The limit case, $\varphi_{0}(x)=x$, generates the arithmetic mean.

Exponential means joint with the arithmetic mean are the only quasiarithmetic means that are stable for translations.

Definition 4.2 Given $\alpha \neq 0$, the exponential mean $A_{\alpha}$ is the aggregation function defined by

$$
A_{\alpha}(\boldsymbol{x})=\frac{1}{\alpha} \ln \frac{e^{\alpha x_{1}}+\cdots+e^{\alpha x_{n}}}{n} .
$$

Proposition 4.3 For every $\alpha \neq 0, A_{\alpha}$ is continuous, idempotent, symmetric, strictly monotonic, compensative, stable for translations, invariant for replications and decomposable. In addition, $A_{\alpha}$ is $S$-convex for every $\alpha>0$, and $S$-concave for every $\alpha<0$.

Proof. Continuity, idempotency, symmetry, strict monotonicity, decomposability and stability for translations are well-known properties of exponential means, as mentioned above. Invariance for replications is straightforward. It is routinary to see that $A_{\alpha}$ is convex if $\alpha>0$, and concave 
if $\alpha<0$. Then, by symmetry, $A_{\alpha}$ is $S$-convex for every $\alpha>0$, and $S$-concave for every $\alpha<0$ (see Marshall and Olkin [12, Chapter 3, Prop. C.2]).

Given $\alpha \neq 0$, the dual of $A_{\alpha}$ is the aggregation function $A_{\alpha}^{*}$ defined by

$$
A_{\alpha}^{*}(\boldsymbol{x})=-\frac{1}{\alpha} \ln \frac{e^{-\alpha x_{1}}+\cdots+e^{-\alpha x_{n}}}{n},
$$

i.e., $A_{\alpha}^{*}=A_{-\alpha}$.

Proposition 4.4 For every $\alpha \neq 0, A_{\alpha}^{*}$ is continuous, idempotent, symmetric, strictly monotonic, compensative, stable for translations, invariant for replications and decomposable. In addition, $A_{\alpha}^{*}$ is $S$-concave for every $\alpha>0$, and $S$-convex for every $\alpha<0$.

Proof. By $A_{\alpha}^{*}=A_{-\alpha}$ and Proposition 4.3.

Given $\alpha \neq 0$, the self-dual core of $A_{\alpha}$ is the aggregation function $\widehat{A}_{\alpha}$ defined by

$$
\widehat{A}_{\alpha}(\boldsymbol{x})=\frac{1}{2 \alpha} \ln \frac{e^{\alpha x_{1}}+\cdots+e^{\alpha x_{n}}}{e^{-\alpha x_{1}}+\cdots+e^{-\alpha x_{n}}} .
$$

Proposition 4.5 For every $\alpha \neq 0, \widehat{A}_{\alpha}$ is continuous, idempotent, symmetric, strictly monotonic, compensative, stable for translations, self-dual and invariant for replications.

Proof. It is a direct consequence of Propositions 3.5 and 4.3 .

It is important to note that $\widehat{A}_{\alpha}$ is not decomposable (see García-Lapresta and Marques Pereira [9, Remark 37]).

Given $\alpha \neq 0$, the anti-self-dual remainder of $A_{\alpha}$ is the mapping $\widetilde{A}_{\alpha}$ defined by

$$
\widetilde{A}_{\alpha}(\boldsymbol{x})=\frac{1}{2 \alpha} \ln \frac{\left(e^{\alpha x_{1}}+\cdots+e^{\alpha x_{n}}\right)\left(e^{-\alpha x_{1}}+\cdots+e^{-\alpha x_{n}}\right)}{n^{2}} .
$$

Proposition 4.6 For every $\alpha \neq 0, \widetilde{A}_{\alpha}(\boldsymbol{x})=0$ if and only if $x_{1}=\cdots=x_{n}$. Moreover, $\widetilde{A}_{\alpha}$ is continuous, symmetric, anti-self-dual, invariant for translations and invariant for replications. In addition, $\widetilde{A}_{\alpha}$ is $S$-convex for every $\alpha>0$, and $S$-concave for every $\alpha<0$.

Proof. It is a direct consequence of Propositions 3.8, 3.9 and 4.3, by the fact that $\widetilde{A}_{\alpha}(\boldsymbol{x})=0$, i.e., $\sum_{i, j=1}^{n} e^{x_{i}-x_{j}}=n^{2}$, implies $x_{1}=\cdots=x_{n}$; and because $\widetilde{A}_{\alpha}$ is convex for every $\alpha>0$, and concave for every $\alpha<0$ (using the same arguments that in Proposition 4.3). 
Remark 4.7 If $\alpha>0$, then $0 \leq \widetilde{A}_{\alpha}(\boldsymbol{x}) \leq 0.5$ for every $\boldsymbol{x} \in[0,1]^{n}$. If $\alpha<0$, then $-0.5 \leq \widetilde{A}_{\alpha}(\boldsymbol{x}) \leq 0$ for every $\boldsymbol{x} \in[0,1]^{n}$.

The following result presents the limits of the exponential means and the associated dual, selfdual-core and anti-self-dual remainder. The proof is by straightforward application of l'Hospital's rule (see García-Lapresta and Marques Pereira [9]).

Proposition 4.8 The following statements hold:

$$
\begin{aligned}
& \text { 1. } \lim _{\alpha \rightarrow \infty} A_{\alpha}(\boldsymbol{x})=x^{*} \text {. } \\
& \text { 2. } \lim _{\alpha \rightarrow-\infty} A_{\alpha}(\boldsymbol{x})=x_{*} \text {. }
\end{aligned}
$$

3. $\lim _{\alpha \rightarrow 0} A_{\alpha}(\boldsymbol{x})=\frac{x_{1}+\cdots+x_{n}}{n}$.

4. $\lim _{\alpha \rightarrow \infty} A_{\alpha}^{*}(\boldsymbol{x})=x_{*}$.

5. $\lim _{\alpha \rightarrow-\infty} A_{\alpha}^{*}(\boldsymbol{x})=x^{*}$.

6. $\lim _{\alpha \rightarrow 0} A_{\alpha}^{*}(\boldsymbol{x})=\frac{x_{1}+\cdots+x_{n}}{n}$.

7. $\lim _{\alpha \rightarrow \infty} \widehat{A}_{\alpha}(\boldsymbol{x})=\lim _{\alpha \rightarrow-\infty} \widehat{A}_{\alpha}(\boldsymbol{x})=\frac{x_{*}+x^{*}}{2}$.

8. $\lim _{\alpha \rightarrow 0} \widehat{A}_{\alpha}(\boldsymbol{x})=\frac{x_{1}+\cdots+x_{n}}{n}$.

9. $\lim _{\alpha \rightarrow \infty} \widetilde{A}_{\alpha}(\boldsymbol{x})=\frac{x^{*}-x_{*}}{2}$.

10. $\lim _{\alpha \rightarrow-\infty} \widetilde{A}_{\alpha}(\boldsymbol{x})=-\frac{x^{*}-x_{*}}{2}$.

11. $\lim _{\alpha \rightarrow 0} \widetilde{A}_{\alpha}(\boldsymbol{x})=0$. 


\section{Poverty measures}

We consider a population consisting of $n$ individuals, with $n \geq 2$. An income distribution is represented by a vector $\boldsymbol{x}=\left(x_{1}, \ldots, x_{n}\right) \in[0, \infty)^{n}$, where $x_{i}$ is the income of individual $i$.

Since Sen [14], any poverty measure should consist of a method to identify the poor and an aggregative measure. Thus, the first step to define a poverty measure is the identification of the poor people in society. This step requires the specification of a poverty line $z \in(0, \infty)$, which represents the necessary income to maintain a minimum level of living. For an income distribution $\boldsymbol{x}$, person $i$ is considered as poor if $x_{i}<z$. Otherwise he is non-poor or rich.

We denote the set of poor people by

$$
Q(\boldsymbol{x}, z)=\left\{i \in\{1, \ldots, n\} \mid x_{i}<z\right\},
$$

and by $q(\boldsymbol{x}, z)$ the number of the poor, i.e., $q(\boldsymbol{x}, z)=\# Q(\boldsymbol{x}, z)$.

Once the poor people are identified, the second stage to determine the extent of the poverty is the aggregation step. In what follows, a poverty measure is a non-constant function $P(\boldsymbol{x}, z)$ of the income distribution $\boldsymbol{x}$ and the poverty line $z$.

\subsection{Axioms}

A number of axioms are usually assumed for a poverty measure.

- Poverty Focus (PF): For all $\boldsymbol{x}, \boldsymbol{y} \in[0, \infty)^{n}, z \in(0, \infty)$ and $i \in\{1, \ldots, n\}$, if $Q(\boldsymbol{x}, z)=$ $Q(\boldsymbol{y}, z)=Q, i \in Q$ and $x_{i}=y_{i}$, then $P(\boldsymbol{x}, z)=P(\boldsymbol{y}, z)$.

- Poverty Monotonicity (PM): For all $\boldsymbol{x}, \boldsymbol{y} \in[0, \infty)^{n}, z \in(0, \infty)$ and $i \in\{1, \ldots, n\}$, if $Q(\boldsymbol{x}, z)=Q(\boldsymbol{y}, z)=Q, i \in Q, x_{i}>y_{i}$ and $x_{j} \neq y_{j}$ for every $j \in\{1, \ldots, n\} \backslash\{i\}$, then $P(\boldsymbol{x}, z)<P(\boldsymbol{y}, z)$.

Since poverty measurement is concerned with the deprivations of the poor people, these two properties, postulated by Sen [14], are considered as basic axioms for a poverty measure. Thus, PF requires that a poverty index should not depend on the non-poor people incomes: the poverty level does not vary if the non-poor incomes change as long as the poor set remains unchanged. PM demands that poverty should increase if the income of a poor person decreases.

The following axiom is concerned with inequality among the poor. In the inequality field, the Pigou-Dalton transfer principle establishes that a transfer from a richer person to a poorer one decreases inequality. Sen [14] introduces the counterpart of this principle in the poverty field. We begin with a definition. 
Given $\boldsymbol{x}, \boldsymbol{y} \in[0, \infty)^{n}$, we say that $\boldsymbol{y}$ is obtained from $\boldsymbol{x}$ by a progressive transfer among the poor if for two poor individuals $i, j \in Q(\boldsymbol{x}, z)$ there exists $h>0$ such that $x_{i}<x_{j}$, $y_{i}=x_{i}+h \leq x_{j}-h=y_{j}$ and $y_{k}=x_{k}$ for every $k \in\{1, \ldots, n\} \backslash\{i, j\}$. In other words, a poor person gives an amount of income to a poorer one. According to the Pigou-Dalton principle, inequality among the poor should decrease under a progressive transfer. Then the Tranfer Sensitivity axiom below requires poverty also to decrease.

- Transfer Sensitivity (TS): For all $\boldsymbol{x}, \boldsymbol{y} \in[0, \infty)^{n}$ and $z \in(0, \infty)$, if $\boldsymbol{y}$ is obtained from $\boldsymbol{x}$ by a progressive transfer among the poor, then $P(\boldsymbol{y}, z)<P(\boldsymbol{x}, z)$.

A progressive transfer among the poor entails an increment of income for one poor individual, and a decrement for other poor person, the richer of the two. This TS axiom goes beyond PM and demands that greater weight should be placed on the poorer person and poverty should decrease if inequality among the poor decreases.

A normalization condition is also usually assumed. This property requires that if all the individuals are non-poor, then the society deprivation level is equal to 0 .

- Normalization $(\mathbf{N})$ : For all $\boldsymbol{x}, \boldsymbol{y} \in[0, \infty)^{n}$ and $z \in(0, \infty), P(\boldsymbol{x}, z)=0$ if and only if $Q(\boldsymbol{x}, z)=\emptyset$, i.e., $x_{i} \geq z$ for every $i \in\{1, \ldots, n\}$.

The two following invariance axioms are also standard requirements for a poverty measure:

- Poverty Symmetry (PS): For all $\boldsymbol{x} \in[0, \infty)^{n}, z \in(0, \infty)$ and permutation $\sigma$ on $\{1, \ldots, n\}$, it holds $P\left(\boldsymbol{x}_{\sigma}, z\right)=P(\boldsymbol{x}, z)$.

- Replication Invariance $(\mathbf{R I})$ : For all $\boldsymbol{x}, \boldsymbol{y} \in[0, \infty)^{n}$ and $z \in(0, \infty)$, if $\boldsymbol{y}$ is obtained from $\boldsymbol{x}$ by a replication, i.e., $\boldsymbol{y}=(\overbrace{\boldsymbol{x}, \ldots, \boldsymbol{x}}^{m})$ for some $m \in \mathbb{N}$, then $P(\boldsymbol{y}, z)=P(\boldsymbol{x}, z)$.

PS establishes that no other characteristic apart from the income deprivation matters in defining a poverty index. In turn, RI allows us to compare populations of different sizes.

The first poverty measure introduced in the literature is the headcount ratio,

$$
H:[0, \infty)^{n} \times(0, \infty) \longrightarrow[0,1]
$$

defined by

$$
H(\boldsymbol{x}, z)=\frac{q(\boldsymbol{x}, z)}{n},
$$

that measures the percentage of poor people in the society. 
This is a crude index, which is able to capture the incidence of poverty. However, it is able to capture neither the intensity nor the inequality among the poor. In fact it violates both $\mathbf{P M}$ and TS, since it does not change if the income of a poor decreases, and under progressive transfers among the poor.

In most cases, measuring poverty involves gauging the extent of the deprivation felt by each individual, once the income poverty line has been determined. One of the most used procedures to measure individual $i$ 's shortfall is to consider the normalized gap of individual $i$, defined as

$$
g_{i}=\max \left\{\frac{z-x_{i}}{z}, 0\right\} .
$$

Notice that $g_{i} \in[0,1], g_{i}=0 \Leftrightarrow x_{i} \geq z$, and $g_{i}=1 \Leftrightarrow x_{i}=0$.

Moreover, the normalized gaps are invariant under proportional income changes. In other words, the function $G:[0, \infty)^{n} \times(0, \infty) \longrightarrow[0,1]^{n}$ defined by $G(\boldsymbol{x}, z)=\left(g_{1}, \ldots, g_{n}\right)$ is homogeneous of degree 0: $G\left(\lambda x_{i}, \ldots, \lambda x_{n}, \lambda z\right)=G\left(x_{i}, \ldots, x_{n}, z\right)$ for every $\lambda>0$.

On the other hand, a progressive transfer among the poor leads to an increment in the richer individual gap whereas the poorer person gap decreases. Since the richer gap is smaller than the poor one, then the progressive transfers among the poor incomes are equivalent to the progressive transfers among the poor gaps. Then, the TS axiom is to be fulfilled whenever the function is $S$-convex either in incomes or in gaps ${ }^{3}$.

\subsection{The results}

We now introduce the following single parameter class of poverty measures, using the headcount ratio and the exponential means of the normalized gaps of the poor.

Definition 5.1 Given $\alpha>0$, the poverty measure associated with $A_{\alpha}$ is the function

$$
P_{\alpha}:[0, \infty)^{n} \times(0, \infty) \longrightarrow[0,1]^{n}
$$

defined by

$$
P_{\alpha}(\boldsymbol{x}, z)= \begin{cases}H(\boldsymbol{x}, z) \cdot A_{\alpha}\left(g_{1}^{\prime}, \ldots, g_{q}^{\prime}\right)=\frac{q}{n} \cdot \frac{1}{\alpha} \ln \frac{e^{\alpha g_{1}^{\prime}}+\cdots+e^{\alpha g_{q}^{\prime}}}{q}, & \text { if } q \neq 0, \\ 0, & \text { if } q=0,\end{cases}
$$

where $q=q(\boldsymbol{x}, z)$ and $g_{1}^{\prime}, \ldots, g_{q}^{\prime}$ are the non-null elements of $g_{1}, \ldots, g_{n}$.

Proposition 5.2 For every $\alpha>0, P_{\alpha}$ satisfies PF, PM, TS, N, PS, and RI.

\footnotetext{
${ }^{3}$ A classical result (Marshall and Olkin [12, Chapter 4, Proposition A.1]) establishes that $\boldsymbol{x} \succcurlyeq \boldsymbol{y}$ if and only if $\boldsymbol{y}$ can be derived from $\boldsymbol{x}$ by means of a finite sequence of permutations and/or progressive transfers.
} 
Proof. By construction, $P_{\alpha}$ satisfies $\mathbf{P F}$ and $\mathbf{N}$.

PM: By hypothesis, $H(\boldsymbol{x}, z)=H(\boldsymbol{y}, z)$. From $x_{i}>y_{i}$ we have $\frac{z-x_{i}}{z}<\frac{z-y_{i}}{z}$. Then, by the strict monotonicity of $A_{\alpha}$, we obtain $P(\boldsymbol{x}, z)<P(\boldsymbol{y}, z)$.

TS: Given $i, j \in Q(\boldsymbol{x}, z)$ such that $x_{i}<x_{j}, y_{i}=x_{i}+h \leq x_{j}-h=y_{j}$ for some $h>0$, and $y_{k}=x_{k}$ for every $k \in\{1, \ldots, n\} \backslash\{i, j\}$, we have $y_{j}<x_{j}<z$ and $Q(\boldsymbol{x}, z)=Q(\boldsymbol{y}, z)$, hence $H(\boldsymbol{x}, z)=H(\boldsymbol{y}, z)$. By Proposition 4.3, $A_{\alpha}$ is $S$-convex and, consequently, satisfies TS. Then, we have $P(\boldsymbol{y}, z)<P(\boldsymbol{x}, z)$.

PS: Given a permutation $\sigma$ on $\{1, \ldots, n\}$, we have $Q\left(\boldsymbol{x}_{\sigma}, z\right)=Q(\boldsymbol{x}, z)$, hence $H\left(\boldsymbol{x}_{\sigma}, z\right)=$ $H(\boldsymbol{x}, z)$. Taking into account that $A_{\alpha}$ is symmetric, we obtain PS.

RI: It is easy to see that if $\boldsymbol{y}$ is obtained from $\boldsymbol{x}$ by a replication, then $H(\boldsymbol{y}, z)=H(\boldsymbol{x}, z)$. Taking into account that $A_{\alpha}$ satisfies invariance for replications, we obtain $P(\boldsymbol{x}, z)=P(\boldsymbol{y}, z)$.

Proposition 5.2 above establishes that for every $\alpha>0, P_{\alpha}$ satisfies the basic poverty properties. Moreover, since $P_{\alpha}$ is defined directly on normalized income gaps, it is homogeneous of degree 0 . This is an interesting property that ensures that the poverty level does not change when the units in which income is measured change. All the poverty indices referred to as "relative" satisfy this property. In addition, it is easy to show that if the same amount of extra income, say $k$, is given to each poor person as long as the poor set does not change, the poverty level is reduced by $\frac{q}{n} \frac{k}{z}$. This property is also quite interesting, since it provides a starting point to analyze poverty policies.

Among the poverty measures proposed in the literature, only the Gini-type indices such as the Sen index, the Sen modified index, the Thon index and so on (see for instance Chakravarty [4]) fulfill these two properties. However, none of the Gini-type poverty measures are more sensitive to progressive transfers at the lower end than at the upper end of the income profile. This property, often invoked in the inequality and poverty framework, ensures that the measure is focused on the poorest of the poor and may be formalized as follows.

- Diminishing Transfer Sensitivity (DTS): For all $\boldsymbol{x}, \boldsymbol{y} \in[0, \infty)^{n}$ and $z \in(0, \infty)$, if $Q(\boldsymbol{x}, z)=Q(\boldsymbol{y}, z)$ and $\boldsymbol{y}$ is obtained from $\boldsymbol{x}$ by a progressive transfer from the poor person with income $x_{i}+c$ to the poor person with income $x_{i}$, for some $c>0$, then the magnitude of decrease in poverty $P(\boldsymbol{x}, z)-P(\boldsymbol{y}, z)$ is higher the lower $x_{i}$.

This principle demands that more weight should be assigned to transfers to a poor person the poorer the person happens to be. 
Proposition 5.3 For every $\alpha>0, P_{\alpha}$ satisfies DTS and it reduces under a progressive transfer by a larger amount the higher is the value of $\alpha$.

Proof. It is straightforward taking into account the exponential function is increasing and convex.

This proposition enables us to interpret the $\alpha$ parameter as the degree of sensitivity to the poorest. It may be worth noting that

$$
\lim _{\alpha \rightarrow \infty} P_{\alpha}(\boldsymbol{x}, z)=\frac{q}{n} g^{*}=\frac{q}{n} \frac{z-x_{*}}{z} .
$$

Remark 5.4 When $\alpha>0$, the core $\widehat{A}_{\alpha}$ is idempotent, symmetric, strictly monotonic, and stable for translations (see Proposition 10). The strictly monotonicity axiom implies that $\widehat{A}_{\alpha}$ is increasing in the gap of a poor person. The stability for translations means that equal absolute changes in all poor gaps lead to the same absolute change in $\widehat{A}_{\alpha}$. These properties can be regarded as basic properties of a poverty intensity index. Nevertheless, is not $S$-convex, and then it goes against the Pigou-Dalton transfer principle. For this, it suffices to consider two gaps distributions $(g-c, g, \ldots, g, g+c)$ and $(g, g, \ldots, g, g)$. Obviously the second distribution is obtained from the former by a progressive transfer among the poor. Hence the Pigou-Dalton transfer principle would require $\widehat{A}_{\alpha}$ to decrease, however does not change:

$$
\widehat{A}_{\alpha}(g-c, g, \ldots, g, g+c)=\widehat{A}_{\alpha}(g, g, \ldots, g, g)=g .
$$

In turn, the remainder $\widetilde{A}_{\alpha}$ is symmetric, fulfils that $\widetilde{A}_{\alpha}\left(g_{1}, \ldots, g_{q}\right)=0$ if and only if $g_{1}=\cdots=g_{q}$, and Propositions 3.8 and 4.3 ensure that it is $S$-convex, and consequently the Pigou-Dalton transfer principle is satisfied. Hence, we can obtain a direct interpretation of $\widetilde{A}_{\alpha}$ as a measure of inequality among the poor individuals. In addition $\widetilde{A}_{\alpha}$ is invariant for translations (Proposition 4.6), thus it measures inequality from an absolute point of view and remains invariant if the gaps of all the poor are increased by the same amount. However, $\widetilde{A}_{\alpha}$ does not fulfil PM.

The next proposition, which involves the self-dual core and the anti self-dual remainder of $A_{\alpha}$, provides a decomposition of the poverty measure $P_{\alpha}$ in three components: $H, \widehat{A}_{\alpha}$ and $\widetilde{A}_{\alpha}$.

Proposition 5.5 For every $\alpha>0$, the poverty measure $P_{\alpha}$ associated with $A_{\alpha}$ satisfies the following decomposition:

$$
P_{\alpha}(\boldsymbol{x}, z)= \begin{cases}H(\boldsymbol{x}, z) \cdot\left(\widehat{A}_{\alpha}\left(g_{1}^{\prime}, \ldots, g_{q}^{\prime}\right)+\widetilde{A}_{\alpha}\left(g_{1}^{\prime}, \ldots, g_{q}^{\prime}\right)\right), & \text { if } q \neq 0, \\ 0, & \text { if } q=0,\end{cases}
$$

where $q=q(\boldsymbol{x}, z)$ and $g_{1}^{\prime}, \ldots, g_{q}^{\prime}$ are the non-null elements of $g_{1}, \ldots, g_{n}$. 
When all the poor individuals have the same gap and, thus, there is not inequality among the poor, the extent of the poverty is given by the product of the headcount ratio and $\widehat{A}_{\alpha}$. If there are progressive transfers of income among the poor, both $P_{\alpha}$ and $\widetilde{A}_{\alpha}$ are bound to decrease, whereas $\widehat{A}_{\alpha}$ can vary in any way. Therefore, societies with the same proportion of poor individuals, $H$, and the same location measure, $\widehat{A}_{\alpha}$, may be ordered by considering the dispersion of the gap distribution, $\widetilde{A}_{\alpha}$.

On the other hand, the effect of an increment of the gap in a poor individual implies an increase in $P_{\alpha}$ and in $\widehat{A}_{\alpha}$, since both of them satisfy PM. In contrast, the change in $\widetilde{A}_{\alpha}$ depends on the effect of this change for the distribution dispersion. Consequently, societies with the same proportion of poor individuals, $H$, and the same dispersion of the gap distributions $\widetilde{A}_{\alpha}$ may be ordered by considering the location measure, $\widehat{A}_{\alpha}$.

Hence, the three components in the proposition above can be interpreted as follows:

1. $H$ is a measure of the incidence of poverty.

2. The intensity of poverty as the depths of poor individuals in the society is summarized by the location measure $\widehat{A}_{\alpha}$.

3. The inequality of the poor's gap distribution is captured by the dispersion measure, $\widetilde{A}_{\alpha}$, which provides the sensitivity to the inequality among the poor.

According to Sen [14], these three indicators can give an adequate image of poverty.

Similarly to Definition 5.1, it is possible to introduce a family of poverty measures for $\alpha<0$ with the same results (recall that $A_{\alpha}^{*}=A_{-\alpha}$ ).

Definition 5.6 Given $\alpha<0$, the poverty measure associated with $A_{\alpha}$ is the function

$$
P_{\alpha}:[0, \infty)^{n} \times(0, \infty) \longrightarrow[0,1]^{n}
$$

defined by

$$
P_{\alpha}(\boldsymbol{x}, z)= \begin{cases}H(\boldsymbol{x}, z) \cdot A_{\alpha}^{*}\left(g_{1}^{\prime}, \ldots, g_{q}^{\prime}\right)=\frac{q}{n} \cdot \frac{-1}{\alpha} \ln \frac{e^{-\alpha g_{1}^{\prime}}+\cdots+e^{-\alpha g_{q}^{\prime}}}{q}, & \text { if } q \neq 0, \\ 0, & \text { if } q=0,\end{cases}
$$

where $q=q(\boldsymbol{x}, z)$ and $g_{1}^{\prime}, \ldots, g_{q}^{\prime}$ are the non-null elements of $g_{1}, \ldots, g_{n}$.

Proposition 5.7 For every $\alpha<0, P_{\alpha}$ satisfies $\boldsymbol{P F}, \boldsymbol{P M}, \boldsymbol{T S}, \boldsymbol{N}, \boldsymbol{P S}$, and $\boldsymbol{R I}$. 
Proposition 5.8 For every $\alpha<0$, the poverty measure $P_{\alpha}$ associated with $A_{\alpha}$ satisfies the following decomposition:

$$
P_{\alpha}(\boldsymbol{x}, z)= \begin{cases}H(\boldsymbol{x}, z) \cdot\left(\widehat{A}_{\alpha}\left(g_{1}^{\prime}, \ldots, g_{q}^{\prime}\right)-\widetilde{A}_{\alpha}\left(g_{1}^{\prime}, \ldots, g_{q}^{\prime}\right)\right), & \text { if } q \neq 0, \\ 0, & \text { if } q=0,\end{cases}
$$

where $q=q(\boldsymbol{x}, z)$ and $g_{1}^{\prime}, \ldots, g_{q}^{\prime}$ are the non-null elements of $g_{1}, \ldots, g_{n}$.

Remark 5.9 An alternative class of poverty measures can be proposed using the exponentials means of the normalized gap distribution: $A_{\alpha}\left(g_{1}, \ldots, g_{n}\right)$, whenever $\alpha>0$, or $A_{\alpha}^{*}\left(g_{1}, \ldots, g_{n}\right)$, whenever $\alpha<0$. The indices in this families satisfy PF, PM, TS, N, PS, and RI. They are sensitive to the headcount ratio, the intensity, and the inequality among the poor. However, contrary to our proposal, they do not admit a straightforward decomposition in the three basic components $H, \widehat{A}_{\alpha}$ and $\widetilde{A}_{\alpha}$, but only in the two last ones.

\subsection{An illustrative example}

We now illustrate the possibilities of the decomposition of the family proposed in this paper. First, consider eight income distributions displayed in the first column of Table 1. The second column includes their corresponding normalized gaps when the poverty line is equal to 1000 . Notice that distributions $\boldsymbol{x}^{2}, \boldsymbol{x}^{3}, \boldsymbol{x}^{5}, \boldsymbol{x}^{6}, \boldsymbol{x}^{7}$ and $\boldsymbol{x}^{8}$ share the same average income of the poor and the averages of the others two are close to this amount. However their poverty levels are quite different and the decomposition of the poverty index $P_{\alpha}$ in its three contribution components, $H, \widehat{A}_{\alpha}$ and $\widetilde{A}_{\alpha}$, displayed in the last four columns of the Table 1 for $\alpha=1$, allows us to determine where the differences stem from. For instance, notice that $\boldsymbol{x}^{1}$ and $\boldsymbol{x}^{2}$ have the same inequality and the same intensity, and the difference in their poverty levels arise from the different percentages of poor people. Distributions $\boldsymbol{x}^{2}$ and $\boldsymbol{x}^{3}$ have the same headcount ratio and the poverty intensity level, nevertheless income among the poor are more equally distributed in $\boldsymbol{x}^{2}$ than in $\boldsymbol{x}^{3}$. By contrast, $\boldsymbol{x}^{3}$ and $\boldsymbol{x}^{4}$ share the headcount ratio and the inequality levels, being different their poverty intensities.

In general, we may compare any pair of distributions and analyze its poverty components to better understand their differences. For example, if we concentrate on distributions $\boldsymbol{x}^{6}, \boldsymbol{x}^{7}$ and $\boldsymbol{x}^{8}$, we may conclude that $\boldsymbol{x}^{6}$ exhibits the lowest headcount ratio, while $\boldsymbol{x}^{7}$ and $\boldsymbol{x}^{8}$ have the lowest values of inequality and intensity respectively.

As already mentioned, all the measures in the poverty family $P_{\alpha}$ fulfils DTS. Distributions $\boldsymbol{x}^{2}, \boldsymbol{x}^{3}$ and $\boldsymbol{x}^{5}$ help us to better understand the meaning of this principle. Notice that both $\boldsymbol{x}^{2}$ and $\boldsymbol{x}^{5}$ may be obtained from $\boldsymbol{x}^{3}$ by a progressive transfer. The richest of the poor is the 
donor in the two transfers. The receivers are the poorest person and the second poorest person respectively. The main point is that whereas in the first case the receiver is the poorest person, in the last case is the second poorest. DTS guarantees that the decrease in poverty is greater under the first transfer.

Table 1: Indices values for $\alpha=1$.

\begin{tabular}{llllll}
\hline Incomes & $z=1000$ & $P_{\alpha}$ & $H$ & $\widehat{A}_{\alpha}$ & $\widetilde{A}_{\alpha}$ \\
\hline $\boldsymbol{x}^{1}=(170,500,729,605)$ & $g^{1}=(0.830,0.500,0.271,0.395)$ & 0.521 & 1.000 & 0.500 & 0.021 \\
$\boldsymbol{x}^{2}=(250,500,750,1300)$ & $g^{2}=(0.750,0.500,0.250,0.000)$ & 0.391 & 0.750 & 0.500 & 0.021 \\
$\boldsymbol{x}^{3}=(150,500,850,1300)$ & $g^{3}=(0.850,0.500,0.150,0.000)$ & 0.405 & 0.750 & 0.500 & 0.040 \\
$\boldsymbol{x}^{4}=(330,350,940,2800)$ & $g^{4}=(0.670,0.650,0.060,0.000)$ & 0.373 & 0.750 & 0.457 & 0.040 \\
$\boldsymbol{x}^{5}=(150,600,750,1300)$ & $g^{5}=(0.850,0.400,0.250,0.000)$ & 0.400 & 0.750 & 0.501 & 0.032 \\
$\boldsymbol{x}^{6}=(217,783,1100,3000)$ & $g^{6}=(0.783,0.217,0.000,0.000)$ & 0.270 & 0.500 & 0.500 & 0.040 \\
$\boldsymbol{x}^{7}=(290,500,600,610)$ & $g^{7}=(0.710,0.500,0.400,0.390)$ & 0.509 & 1.000 & 0.500 & 0.008 \\
$\boldsymbol{x}^{8}=(250,300,950,1400)$ & $g^{8}=(0.750,0.700,0.050,0.000)$ & 0.410 & 0.750 & 0.496 & 0.050 \\
\hline
\end{tabular}

Table 2 highlights the role of $\alpha$. In the comparison of $\boldsymbol{x}^{3}$ and $\boldsymbol{x}^{8}$, it can be noted that whereas for $\alpha=1$ distribution $\boldsymbol{x}^{3}$ displays less poverty, when $\alpha=4$ the situation is just the opposite, since more weight is assigned to the poorest of the population. In other words, the greater the value of $\alpha$ the more sensitive to the poorest incomes. In addition, with respect to DTS, when $\alpha=4$ in the same cases mentioned above, the decrease in poverty is even greater.

Table 2: Indices values for $\alpha=4$.

\begin{tabular}{llllll}
\hline Incomes & $z=1000$ & $P_{\alpha}$ & $H$ & $\widehat{A}_{\alpha}$ & $\widetilde{A}_{\alpha}$ \\
\hline $\boldsymbol{x}^{1}=(170,500,729,605)$ & $g^{1}=(0.830,0.500,0.271,0.395)$ & 0.593 & 1.000 & 0.512 & 0.081 \\
$\boldsymbol{x}^{2}=(250,500,750,1300)$ & $g^{2}=(0.750,0.500,0.250,0.000)$ & 0.433 & 0.750 & 0.500 & 0.077 \\
$\boldsymbol{x}^{3}=(150,500,850,1300)$ & $g^{3}=(0.850,0.500,0.150,0.000)$ & 0.482 & 0.750 & 0.500 & 0.142 \\
$\boldsymbol{x}^{4}=(330,350,940,2800)$ & $g^{4}=(0.670,0.650,0.060,0.000)$ & 0.427 & 0.750 & 0.431 & 0.138 \\
$\boldsymbol{x}^{5}=(150,600,750,1300)$ & $g^{5}=(0.850,0.400,0.250,0.000)$ & 0.474 & 0.750 & 0.517 & 0.116 \\
$\boldsymbol{x}^{6}=(217,783,1100,3000)$ & $g^{6}=(0.783,0.217,0.000,0.000)$ & 0.317 & 0.500 & 0.500 & 0.134 \\
$\boldsymbol{x}^{7}=(290,500,600,610)$ & $g^{7}=(0.710,0.500,0.400,0.390)$ & 0.537 & 1.000 & 0.504 & 0.032 \\
$\boldsymbol{x}^{8}=(250,300,950,1400)$ & $g^{8}=(0.750,0.700,0.050,0.000)$ & 0.475 & 0.750 & 0.463 & 0.170 \\
\hline
\end{tabular}




\section{Concluding remarks}

In literature on economics there exists a well established tradition of aggregating different economic indicators for the purpose of comparing societies or countries. In this issue, we have considered poverty measures associated with exponential means, the only family of quasiarithmetic means satisfying stability for translations.

The transformation from non negative income values $x$ to unit interval valued income gaps $g$ is central in the paper. As usual, it is obtained by means of a threshold $z$ (which defines the subset or coalition of the poor individuals) plus a normalization factor. In the future, we intend to consider the possibility of extending the concept of coalition of the poor, by assigning to each coalition of individuals a Choquet capacity measuring the ratio of poor individuals it contains, on the basis of some unit valued individual degree of poverty (in the present model an individual is either poor, with poverty degree 1 , or non-poor, with poverty degree 0 ; but this depends strongly on the exact poverty threshold). The Choquet capacity value of each coalition would then constitute a generalized (and possibly non additive) measure of the headcount ratio $H$.

\section{Acknowledgments}

García-Lapresta gratefully acknowledges the funding support of the Spanish Ministerio de Ciencia e Innovación (Project ECO2009-07332), ERDF and Junta de Castilla y León (Consejería de Educación, Projects VA092A08 and GR99). Lasso de la Vega and Urrutia gratefully acknowledge the funding support of the Spanish Ministerio de Ciencia e Innovación (Project ECO2009-11213), and the Basque Departamento de Educación e Investigación (Project GIC07/146-IT-377-07).

\section{References}

[1] G. Beliakov, A. Pradera and T. Calvo, Aggregation Functions: A Guide for Practicioners (Springer, Heidelberg, 2007).

[2] T. Calvo, A. Kolesárova, M. Komorníková and R. Mesiar, "Aggregation operators: Properties, classes and construction methods", in Aggregation Operators: New Trends and Applications, eds. T. Calvo, G. Mayor and R. Mesiar (Physica-Verlag, Heidelberg, 2002) pp. 3-104.

[3] S.R. Chakravarty, Ethical Social Index Numbers (Springer-Verlag, New York, 1990).

[4] S.R. Chakravarty, Inequality, Polarization and Poverty: Advances in Distributional Analysis (Springer-Verlag, New York, 2009). 
[5] S.R. Chakravarty and P. Muliere, Welfare indicators: A review and new perspectives. 1. Measurement of inequality, Metron - International Journal of Statistics 61 (2003) 457-497.

[6] S.R. Chakravarty and P. Muliere, Welfare indicators: A review and new perspectives. 2. Measurement of poverty, Metron - International Journal of Statistics 62 (2004) 247-281.

[7] C. Dagum, On the relationship between income inequality measures and social welfare functions, Journal of Econometrics 43 (1990) 91-102.

[8] J. Fodor and M. Roubens, Fuzzy Preference Modelling and Multicriteria Decision Support (Kluwer Academic Publishers, Dordrecht, 1994).

[9] J.L. García-Lapresta and R.A. Marques Pereira, The self-dual core and the anti-self-dual remainder of an aggregation operator, Fuzzy Sets and Systems 159 (2008) 47-62.

[10] M. Grabisch, J.L. Marichal, R. Mesiar and E. Pap, Aggregation Functions (Cambridge University Press, Cambridge, 2009).

[11] A. Kolmogoroff, Sur la notion de la moyenne, Atti della R. Academia Nazionale del Lincei. Rendiconti della Classe di Scienze Fisiche, Mathematiche e Naturali (6) 12 (1930) 388-391.

[12] A.W. Marshall and I. Olkin, Inequalities: Theory of Majorization and Its Applications (Mathematics in Science and Engineering, vol 143, Academic Press, New York, 1972).

[13] M. Nagumo, Über eine Klasse der Mittelwerte, Japanese Journal of Mathematics 7 (1930) $71-79$.

[14] A.K. Sen, Poverty: An ordinal approach to measurement, Econometrica 44 (1976) 219-231.

[15] A.K. Sen, Inequality Reexamined (Oxford University Press, Oxford, 1992). 